\title{
Editorial
}

\section{Filtering and Control for Unreliable Communication 2015}

\author{
Guoliang Wei, ${ }^{1}$ Lifeng $\mathrm{Ma}^{2}$ Zidong Wang, ${ }^{3,4}$ Derui Ding, ${ }^{1}$ and Jun $\mathrm{Hu}^{5,6}$ \\ ${ }^{1}$ Shanghai Key Lab of Modern Optical System, School of Optical-Electrical and Computer Engineering, \\ University of Shanghai for Science and Technology, No. 516, Jungong Road, Shanghai 200093, China \\ ${ }^{2}$ School of Automation, Nanjing University of Science and Technology, Nanjing, Jiangsu 210094, China \\ ${ }^{3}$ Department of Computer Science, Brunel University London, Uxbridge, Middlesex UB8 3PH, UK \\ ${ }^{4}$ Faculty of Engineering, King Abdulaziz University, Jeddah 21589, Saudi Arabia \\ ${ }^{5}$ Department of Applied Mathematics, Harbin University of Science and Technology, Harbin 150080, China \\ ${ }^{6}$ Research Institute of Intelligent Control and Systems, Harbin Institute of Technology, Harbin 150001, China
}

Correspondence should be addressed to Guoliang Wei; guoliang.wei1973@gmail.com

Received 4 October 2015; Accepted 4 October 2015

Copyright (C) 2015 Guoliang Wei et al. This is an open access article distributed under the Creative Commons Attribution License, which permits unrestricted use, distribution, and reproduction in any medium, provided the original work is properly cited.

In the past decades, communication networks have been extensively employed in many practical control systems, such as manufacturing plants, aircraft, and spacecraft to transmit information and control signals between the system components. When a control loop is closed via a serial communication channel, a networked control system (NCS) is formed. NCSs have become very popular for their great advantages over traditional systems (e.g., low cost and reduced weight and power requirements). Generally, it has been implicitly assumed that the communication between the system components is perfect; that is, the signals transmitted from the plant always arrive at the filter or controller without any information loss. Unfortunately, such an assumption is not always true. For example, a common feature of the NCSs is the presence of significant network-induced delays and data losses across the networks. Therefore, an emerging research topic that has recently drawn much attention is how to cope with the effect of network-induced phenomena due to the unreliability of the network communication. This special issue aims to bring together the latest approaches to filtering and control as well as fault diagnosis and detection for various systems under unreliable communication. Potential topics in this special issue include (a) filtering or control in stochastic system, (b) network-induced phenomena, (c) fault diagnosis and detection, (d) intelligent algorithm, and (e) applications in real-world systems.

We have solicited submissions to this special issue from control engineers, electrical engineers, computer scientists, and mathematicians. After a rigorous peer review process, the high-quality papers have been selected that provide overviews, solutions, or early promises, to manage, analyze, and interpret dynamical behaviors of various systems. These papers have covered both the theoretical and practical aspects of various systems in the broad areas of dynamical systems, mathematics, statistics, computer science, and practical engineering.

In light of the rapid deployment of sensor network, this special issue starts with a survey paper entitled "A Survey on Multisensor Fusion and Consensus Filtering for Sensor Networks" by W. Li et al., where the focus is to provide a timely review on the two fascinating subjects in the research of sensor networks: multisensor fusion and consensus filtering. The survey covers both classic results and recent advances developed in these two topics. First, some important results in the development of multisensor fusion technology are introduced, with special attention to the fusion with unknown correlations, which ubiquitously exist in most of distributed filtering problems. Next, a systematic review on several widely used consensus filtering approaches is also given. At last, some latest progress and several potential future research directions are outlined.

In recent years, stochastic systems have gained much research interest, and many researchers have extended the existing methodology from deterministic systems to the stochastic ones. To be specific, in the paper entitled "Losslessness of Nonlinear Stochastic Discrete-Time Systems" by 
$\mathrm{X}$. Liu et al., the stochastic losslessness theory of nonlinear stochastic discrete-time systems formulated by the Ito-type difference equations is addressed, and a necessary and sufficient condition is established. Based on the stochastic lossless theory, it is shown that a nonlinear stochastic discrete-time system can be lossless via state feedback, and the condition is developed when a system is feedback equivalent to a lossless system. The $\mathscr{H}_{-}$index for stochastic linear discrete-time systems is discussed in " $\mathscr{H}$ _ Index for Stochastic Linear DiscreteTime Systems" by Y. Li et al. The definition of the $\mathscr{H}_{-}$index is successfully extended to the stochastic systems. A necessary and sufficient condition of $\mathscr{H}_{-}$index is given for such systems in finite horizon. The authors prove that when the $\mathscr{H}_{-}$index is greater than a given value, the feasibility of $\mathscr{H}_{-}$index is equivalent to the solvability of a constrained difference equation. It also points out that the above result can be applied to the fault detection observer design. Finally, some examples are presented to illustrate the proposed theoretical results. In the paper entitled "Robustness Analysis of Hybrid Stochastic Neural Networks with Neutral Terms and TimeVarying Delays" by C. Wu et al., the problem of the robustness of global exponential stability of hybrid stochastic neural networks subject to neutral terms and time-varying delays is addressed. The upper bounds of contraction coefficients of neutral terms and time-varying delays are characterized by using the transcendental equation. Moreover, the following result is proved theoretically: for any globally exponentially stable hybrid stochastic neural networks, if the condition that additive neutral terms and time-varying delays are smaller than the upper bounds is met, then the perturbed neural networks are also guaranteed to be globally exponentially stable. Finally, a numerical simulation example is given to illustrate the presented criteria.

During the past decades, the fault diagnosis and detection theory has received considerable attention, and many remarkable achievements have been obtained. In the paper entitled "Study on Wireless Network Communication in Stage Hydraulic Monitoring System Based on Internet of Things" by Y. Dong et al., a novel stage hydraulic monitoring system is proposed based on Internet of Things (IoT), which is a flexible working method that can save the cost with the low power consumption, high safety, and large scale network, compared with the traditional wired system. In addition, the fault detection and diagnosis process is concerned, which is facilitated by measuring pressure of flow. The failure may occur when the monitored data exceeds the normal range; meanwhile, the maintainers can be informed to ensure that the failure is eliminated in time. Furthermore, the efficiency of monitoring can be improved by use of the wireless sensor network and ZigBee technology. In the paper entitled " $H_{\infty}$ Fault Detection for Linear Discrete Time-Varying Descriptor Systems with Missing Measurements" by G. Deng and H. Zhao, the problem of $H_{\infty}$ fault detection is dealt with for a class of linear discrete time-varying descriptor systems with missing measurements which are characterized by a Bernoulli random binary switching sequence. By translating the $H_{\infty}$ fault detection problem into an indefinite quadratic form problem, a sufficient and necessary condition on the existence of the minimum is derived. Finally, an observer-based $H_{\infty}$ fault detection filter is obtained by solving a matrix differential equation.

Very recently, network science is suffering a rapid development due to its obvious application backgrounds in various fields. As a powerful tool to analyze the networks, graph theory also receives much special attention. In the paper entitled "The Least Algebraic Connectivity of Graphs" by G. Jiang et al., the algebraic connectivity of a graph is defined as the second smallest eigenvalue of the Laplacian matrix of the graph, which is a parameter to measure how well a graph is connected. Two unique graphs are proposed, whose algebraic connectivity attains the minimum among all graphs whose complements are trees, but not stars; moreover, the complements of all graphs are unicyclic graphs, but not stars adding one edge, respectively. In the paper "The Kirchhoff Index of Some Combinatorial Networks" by J.B. Liu et al., the authors investigate the Kirchhoff index of some combinatorial networks. The graph $G^{*}$, constructed from $G$, is the line graph of the subdivision graph $S(G)$. The relationships between $Q_{n}, F Q_{n}$ and its variant networks $\left(Q_{n}\right)^{*},\left(F Q_{n}\right)^{*}$ are established in terms of Kirchhoff index, respectively. In addition, explicit formulae are proposed for expressing the Kirchhoff index of $\left(Q_{n}\right)^{*}$ and $\left(F Q_{n}\right)^{*}$ by making use of the characteristic polynomial of the Laplacian matrix in spectral graph theory.

The filtering problem has been a mainstream research topic in the control theory, and much effort has been made in all kinds of fields such as signal processing, econometrics communication, and control of vehicles. Kalman filtering and $H_{\infty}$ filtering, specially, have attracted much research interests due to their respective advantages. In the paper entitled "Kalman Filtering for Discrete Stochastic Systems with Multiplicative Noises and Random Two-Step Sensor Delays" by $\mathrm{D}$. Chen et al., the optimal Kalman filtering problem is concerned for a class of discrete stochastic systems with multiplicative noises and random two-step sensor delays. To characterize the random phenomena, three Bernoulli distributed random variables with known conditional probabilities are introduced. Based on the state augmentation approach and innovation analysis technique, an optimal Kalman filter is designed in the sense of the minimum mean square error. In addition, in the paper entitled "Study on a Twice Transfer Alignment Based on Dual Model" by S. Chen et al., a twice transfer alignment algorithm based on dual models is presented, which combines the advantages of nonlinear and linear error models. The quaternion matching based on quaternion error model along with extended Kalman filter is used to dispose of the large misalignment in the first phase. Then, in the second transfer alignment, velocity plus attitude matching and classical Kalman filter is adopted. In the paper entitled "Robust Distributed $H_{\infty}$ Filtering for Nonlinear Systems with Sensor Saturations and Fractional Uncertainties with Digital Simulation" by D. Liu et al., the attention is focused on the robust distributed $H_{\infty}$ filtering problem for nonlinear systems subject to sensor saturations and fractional parameter uncertainties. A sufficient condition is derived for the filtering error system to reach the required $H_{\infty}$ performance in terms of recursive linear matrix inequality method. An iterative algorithm is 
then proposed to obtain the filter parameters recursively by solving the corresponding linear matrix inequality. Finally, a numerical example is presented to show the effectiveness of the proposed method.

With the persistent developments in the past forty years, intelligent algorithms have played an irreplaceable important role in control, computer science, and other areas because of their specific superiority compared to the traditional ones. In view of the SVM classification for the imbalanced sand-dust storm data sets, a hybrid self-adaptive sampling method named SRU-AIBSMOTE algorithm is proposed in "Imbalanced Data Sets Classification Based on SVM for Sand-Dust Storm Warning" by Y. Xie et al. This method can adaptively adjust neighboring selection strategy based on the internal distribution of sample sets. It produces virtual minority class instances through randomized interpolation in the spherical space which consists of minority class instances and their neighbors. The random undersampling is also applied to undersample the majority class instances for removal of redundant data in the sample sets. The comparative experimental results on some real data sets show that the SRU-AIBSMOTE method can obtain better classification performance than some traditional classification methods. The bankruptcy prediction problem is studied by using some hybrid intelligence algorithms in "A New Hybrid Algorithm for Bankruptcy Prediction Using Switching Particle Swarm Optimization and Support Vector Machines" by Y. Lu et al.; in particular, a recently developed SPSO algorithm is exploited to search the optimal parameter values of radial basis function (RBF) kernel of the SVM. This new algorithm can largely improve the explanatory power and the stability of the SVM. The proposed algorithm is successfully applied in the bankruptcy prediction problem, where experiment data sets are originally from the UCI Machine Learning Repository and simulation results show the superiority of proposed algorithm over the traditional SVM-based methods combined with genetic algorithm (GA) or the particle swarm optimization (PSO) algorithm alone.

Compared to the above efforts devoted to the fundamental theoretical research, tremendous works also have been carried out in the engineering applications and some excellent achievements have been obtained. The article of "Optimal Design of FPGA Switch Matrix with Ion Mobility Based Nonvolatile ReRAM" by P. Hai-yun and Z. Wengang first explores the mechanism of the ReRAM device based on ion mobility model and develops a physical model based on ion mobility for this device. Some characteristics are investigated to describe the device electrical behavior and then a novel application of the nonvolatile ReRAM is proposed to optimize the design of FPGA switching matrix. Compared with the traditional approach, the results show that it is beneficial to enhance the FPGA performance by using the ReRAM cells for the switching matrix. Based on the Doppler shift, a dynamic navigation method with multisubstations is proposed in "Dynamic Navigation Method with Multisubstations Based on Doppler Shift" by J. Zhao et al.; this paper develops some interesting methods to realize mobile navigation calculation via Doppler shifts. It gives the theory of relationship between the motion parameters (such as directions and speed) and frequency shifts caused by multi base stations. The simulation illustrates the effectiveness of the developed methods. Furthermore, it also gives an application with Google map and dynamical locating position as well as direction on a mobile phone by public wireless network.

\section{Acknowledgments}

This special issue is a timely reflection of the recent research progress in the area of filtering and control for unreliable communication in various systems. We would like to acknowledge all authors for their efforts in submitting highquality papers. We are also very grateful to the reviewers for their thorough and on-time reviews of the papers.

$$
\begin{array}{r}
\text { Guoliang Wei } \\
\text { Lifeng Ma } \\
\text { Zidong Wang } \\
\text { Derui Ding } \\
\text { Jun Hu }
\end{array}
$$




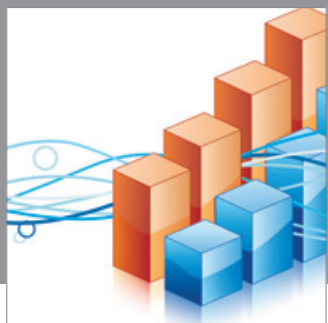

Advances in

Operations Research

mansans

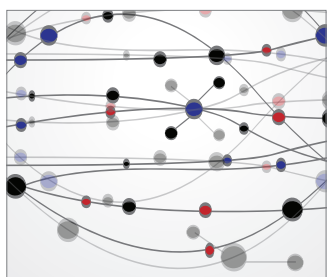

The Scientific World Journal
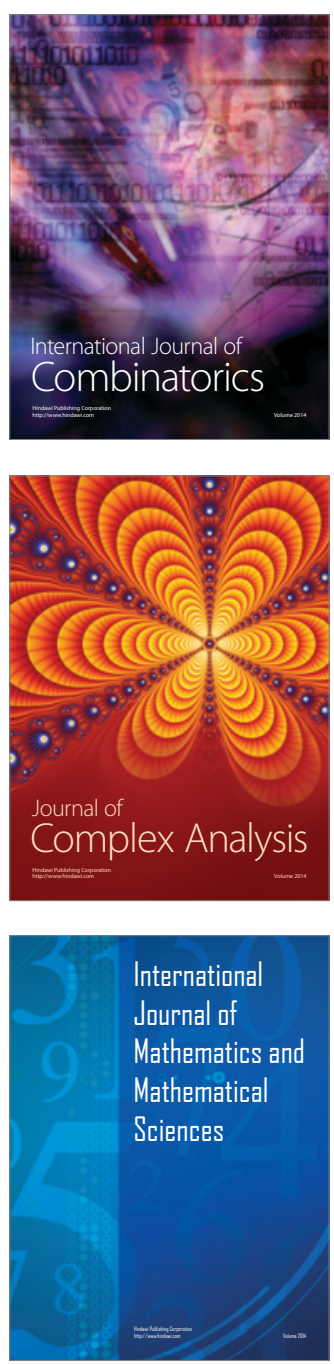
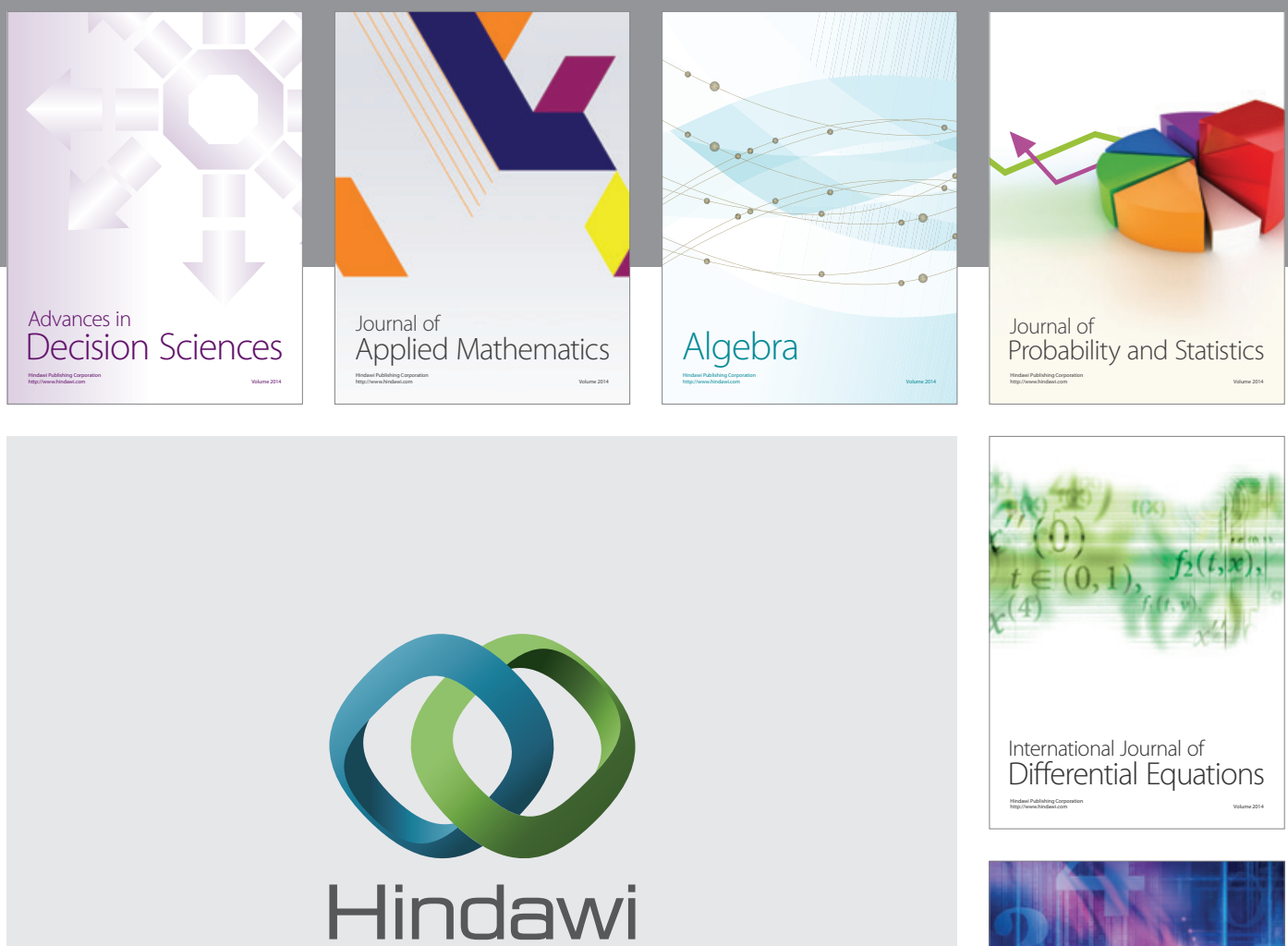

Submit your manuscripts at http://www.hindawi.com
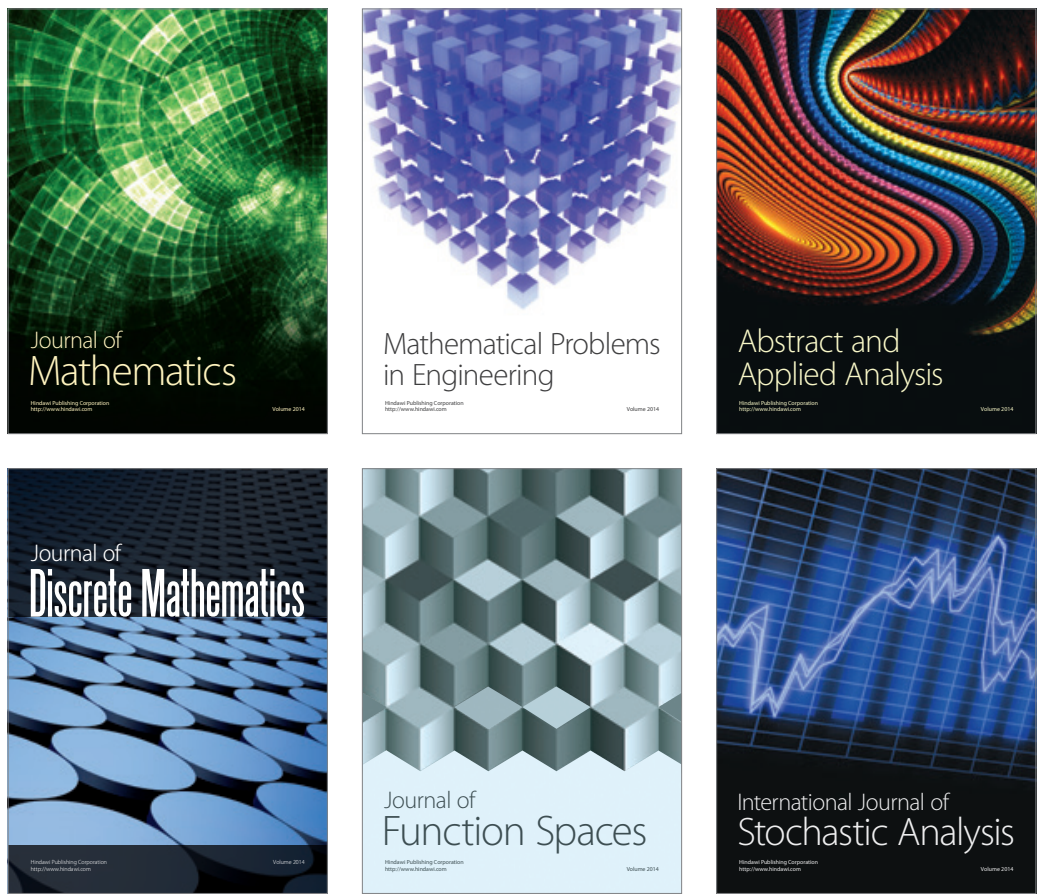

Journal of

Function Spaces

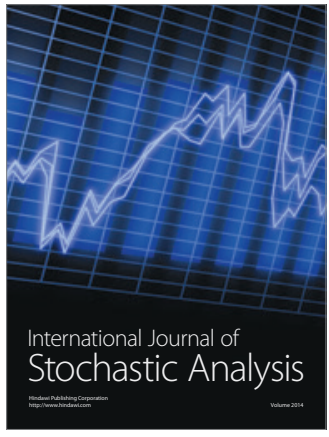

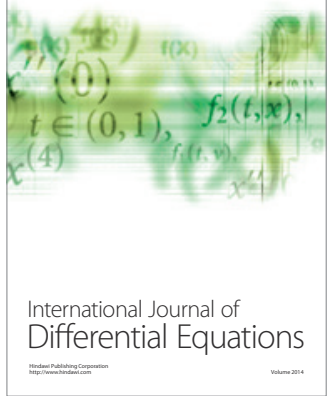
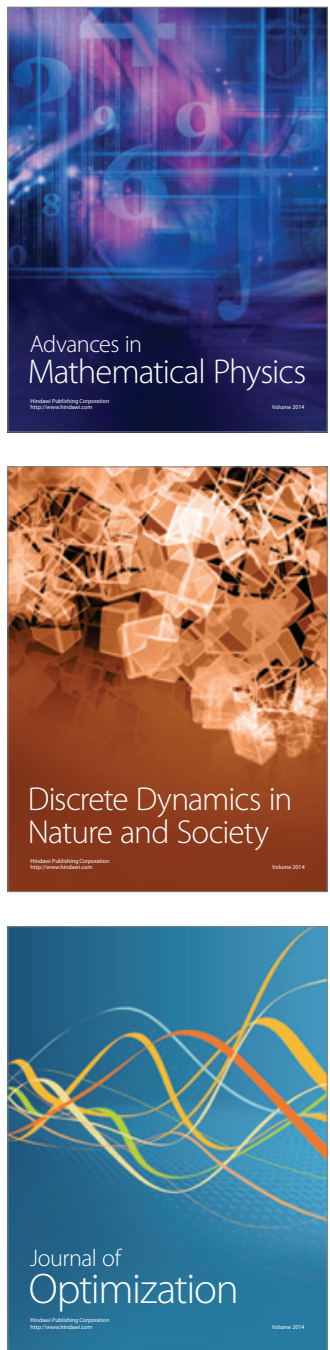